\title{
Singlet-triplet gaps in large multireference systems: Spin-flip-driven alternatives for bioinorganic modeling
}

Aurélien de la Lande, Vicent Moliner, and Olivier Parisel

Citation: The Journal of Chemical Physics 126, 035102 (2007);

View online: https://doi.org/10.1063/1.2423010

View Table of Contents: http://aip.scitation.org/toc/jcp/126/3

Published by the American Institute of Physics

\section{Articles you may be interested in}

General formulation of spin-flip time-dependent density functional theory using non-collinear kernels: Theory, implementation, and benchmarks

The Journal of Chemical Physics 136, 204103 (2012); 10.1063/1.4714499

Spin-flip time dependent density functional theory applied to excited states with single, double, or mixed electron excitation character

The Journal of Chemical Physics 133, 114104 (2010); 10.1063/1.3479401

Multireference quantum chemistry through a joint density matrix renormalization group and canonical transformation theory

The Journal of Chemical Physics 132, 024105 (2010); 10.1063/1.3275806

The radical character of the acenes: A density matrix renormalization group study

The Journal of Chemical Physics 127, 134309 (2007); 10.1063/1.2768362

The spin-flip approach within time-dependent density functional theory: Theory and applications to diradicals The Journal of Chemical Physics 118, 4807 (2003); 10.1063/1.1545679

Perspective: Found in translation: Quantum chemical tools for grasping non-covalent interactions The Journal of Chemical Physics 146, 120901 (2017); 10.1063/1.4978951

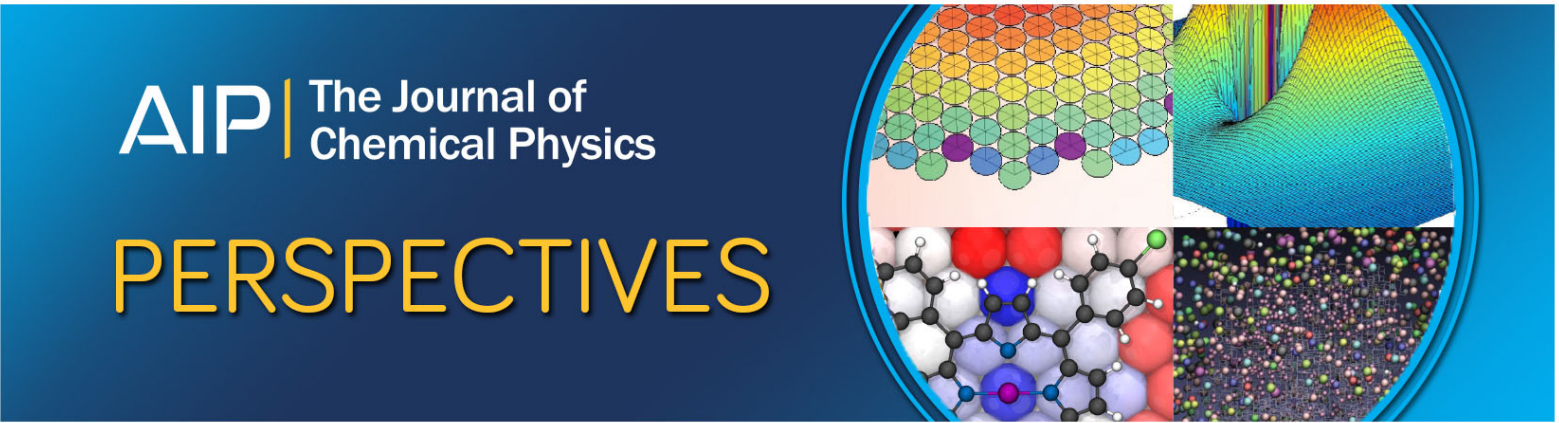




\title{
Singlet-triplet gaps in large multireference systems: Spin-flip-driven alternatives for bioinorganic modeling
}

\author{
Aurélien de la Lande \\ Laboratoire de Chimie Théorique, UMR 7616 CNRS/UPMC, Université Pierre et Marie Curie, Paris 6, \\ Case courrier 137- 4, place Jussieu, F. 75252 Paris Cedex 05, France and Departament de Ciències \\ Experimentals, Universitat Jaume I, Box 224, 12080 Castello, Spain \\ Vicent Moliner \\ Departament de Ciències Experimentals, Universitat Jaume I, Box 224, 12080 Castello, Spain \\ Olivier Parisela) \\ Laboratoire de Chimie Théorique, UMR 7616 CNRS/UPMC, Université Pierre et Marie Curie, Paris 6, \\ Case courrier 137- 4, place Jussieu, F. 75252 Paris Cedex 05, France
}

(Received 10 October 2006; accepted 22 November 2006; published online 17 January 2007)

\begin{abstract}
The proper description of low-spin states of open-shell systems, which are commonly encountered in the field of bioinorganic chemistry, rigorously requires using multireference ab initio methodologies. Such approaches are unfortunately very CPU-time consuming as dynamic correlation effects also have to be taken into account. The broken-symmetry unrestricted (spin-polarized) density functional theory (DFT) technique has been widely employed up to now to bypass that drawback, but despite a number of relative successes in the determination of singlet-triplet gaps, this framework cannot be considered as entirely satisfactory. In this contribution, we investigate some alternative ways relying on the spin-flip time-dependent DFT approach [Y. Shao et al. J. Chem. Phys. 118, 4807 (2003)]. Taking a few well-documented copper-dioxygen adducts as examples, we show that spin-flip (SF)-DFT computed singlet-triplet gaps compare very favorably to either experimental results or large-scale CASMP2 computations. Moreover, it is shown that this approach can be used to optimize geometries at a DFT level including some multireference effects. Finally, a clear-cut added value of the SF-DFT computations is drawn: if pure $a b$ initio data are required, then the electronic excitations revealed by SF-DFT can be considered in designing dramatically reduced zeroth-order variational spaces to be used in subsequent multireference configuration interaction or multireference perturbation treatments.
\end{abstract}

(C) 2007 American Institute of Physics. [DOI: 10.1063/1.2423010]

\section{INTRODUCTION}

Density functional theory ${ }^{1}$ (DFT) has now become the most widely used theoretical approach to deal with large-size molecular systems as it provides correlated results for computational costs comparable to the Hartree-Fock (HF) approach. Taking a large part of dynamic correlation into account, it appears as an alternative to multireference configuration interactions (MRCIs) or multireference perturbations (MRPTs) in some cases and is thus routinely used when modeling organic, organometallic, or inorganic chemistry systems. ${ }^{2-6}$

In principle, DFT can deal with every kind of electronic structures, provided, however, the exact unknown functional would be used. Approximating the exact functional and using the Kohn-Sham (KS) formalism, ${ }^{7}$ as is usually done, intrinsically precludes some systems to be addressed due to the monodeterminantal character of the KS determinant, a

\footnotetext{
${ }^{a)}$ Author to whom correspondence should be addressed.

Electronic mail: parisel@1ct.jussieu.fr; URL: http://www.lct.jussieu.fr; Fax: 0033144274117.
}

feature which, formally, cannot account for nondynamic correlation. Two-electron singlet diradicals, ${ }^{8}$ and more generally any open-shell system not in its highest spin state, are some of the species that cannot, a priori, be properly described this way. Such systems are by far not anecdotic as they are frequently encountered in bioinorganic chemistry. ${ }^{9-12}$ Let us mention, for example, the case of monooxygenase such as dopamine $\beta$-monooxygenase (D $\beta \mathrm{M})$ (Refs. 13-15) or peptidylglycine $\alpha$-hydroxylating monooxygenase (PHM), ${ }^{13-18}$ in which a $\mathrm{Cu}^{\mathrm{I}}$ center interacts with dioxygen to end up with either the high-spin triplet or a low-spin singlet, or tyrosinase, ${ }^{19,20}$ a "torture track" for theory, ${ }^{21}$ in which two $\mathrm{Cu}^{\mathrm{I}}$ centers interact with dioxygen. ${ }^{22-25}$

Using a restricted open-shell DFT formalism allows describing the triplet states of such systems correctly, whereas the description of the singlet states, which might exhibit a more or less pronounced multiconfigurational character, requires, in all-day computations, using an unrestricted formalism (U-DFT) sometimes coupled to broken-symmetry techniques (BSKS), ${ }^{26-31}$ the energy of which are usually corrected. ${ }^{32-34}$ The BSKS procedure can, however, lead to artifacts and its use is still a matter of debates. ${ }^{35-37}$ Moreover, the estimate of the singlet-triplet gaps deduced from BSKS 
computations might sometimes be questionable, or risky, since the nonbroken wave function is sometimes obtained. ${ }^{8,35,38}$

The low- or intermediate-spin states must be monodeterminantal if aiming at using restricted DFT as such although some procedures have been developed to estimate multiplet energies. $^{32,39-44}$ However, there is unfortunately no general way to a priori estimate this monodeterminantal character except, maybe, by comparison to known very closely related species, an hazardous procedure, however. The only way to get an estimate of the single-reference character is to perform dedicated CI, multiconfiguration self-consistent field (MCSCF), or complete active space self-consistent field (CASSCF) calculations. ${ }^{45}$ If the multiconfigurational character is established, then a proper $a b$ initio treatment, if required, should rely on MRPT or MRCI computations. ${ }^{45-47}$ However, such computations are not only difficult to undertake but also too CPU-time consuming: they usually require drastic simplifications of the models investigated, ${ }^{48}$ which can distort the subtle electronic structures involved in bioinorganic processes. ${ }^{49}$

There is thus a real need to have a procedure at hand, even not perfect, capable to provide eventually improvable, but at least reliable, estimates of singlet-triplet gaps for such a priori multireference systems.

In this contribution, we focus on the spin-flip ${ }^{50-53}$ timedependent DFT [SF-(TD)DFT] approach recently proposed by Shao et al., ${ }^{54}$ a procedure further generalized by Wang and Ziegler ${ }^{55,56}$ and will apply this methodology to three complexes relevant to bioinorganic chemistry, namely, $\left.\left[\mathrm{CuCH}(\operatorname{ImH})_{3}\left(\mathrm{O}_{2}\right)\right]^{+}, \quad\left[\mathrm{Cu}(\operatorname{trenMe})_{3}\right)\left(\mathrm{O}_{2}\right)\right]^{+}$, and $\left[\mathrm{CuBH}(\mathrm{Pyra})_{3}\left(\mathrm{O}_{2}\right)\right]$. We, ${ }^{57}$ as other research groups, ${ }^{8,35,36,38,58-61}$ have already investigated these systems which can now be seen as well-documented bioinspired models for the cuprous active sites of the PHM enzyme.

This paper outlines as follows. First, some methodological details are presented (Sec. II). Then, the singlet-triplet gaps are computed and compared to those obtained by other techniques (Sec. III A). Having recognized the reliability of the SF-DFT approach and after a short discussion about spin contamination, we turn to two applications: geometry optimizations (Sec. III C) and the design of zeroth-order multireference variational spaces (Sec. III D).

\section{METHODOLOGY}

\section{A. The spin-flip approach}

The spin-flip (SF) approach aims at describing multideterminantal low-spin states using excitations from a single reference configuration. As illustrated on Fig. 1, top and bottom for $\left[\mathrm{CuCH}(\operatorname{ImH})_{3}\left(\mathrm{O}_{2}\right)\right]^{+}$and $\left.\left[\mathrm{Cu}(\operatorname{trenMe})_{3}\right)\left(\mathrm{O}_{2}\right)\right]^{+}$, respectively, a triplet state-here the $M_{s}=+1$ component-is taken as the reference. Its high-spin character ensures that it is properly described by a single-reference method. $M_{s}=0$ states are obtained by spin-flip excitations of one electron from the reference toward a virtual orbital. The $M_{s}=0 \mathrm{com}-$ ponents of triplet and singlet (close- and open-shell) excited states $^{62}$ can thus be obtained.

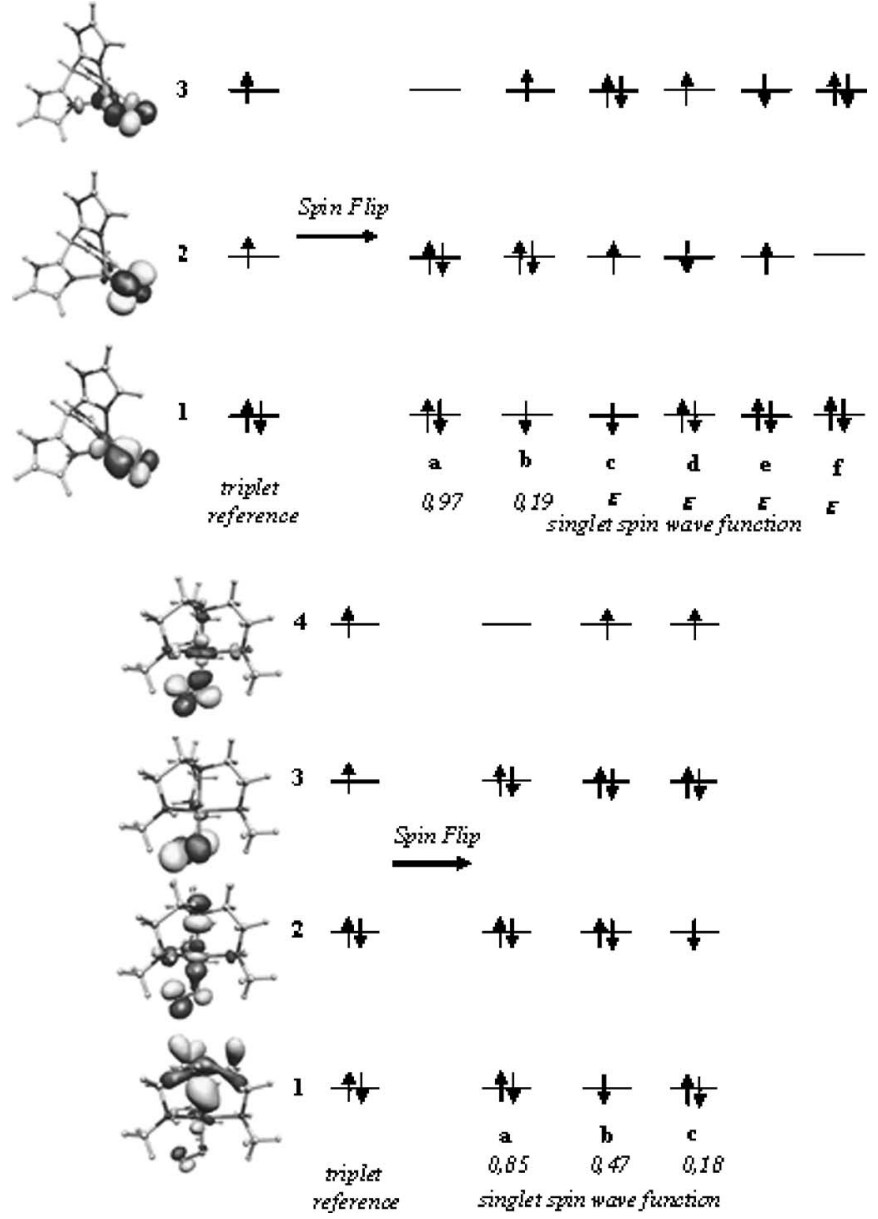

FIG. 1. Illustrations of the spin-flip approach. Top: the four electrons and the three Kohn-Sham molecular orbitals involved in the SF-B3LYP description of the lowest singlet state of the side-on complex $\left[\mathrm{CuCH}(\mathrm{ImH})_{3}\left(\mathrm{O}_{2}\right)\right]^{+}$. Bottom: the six electrons and the four Kohn-Sham molecular orbitals involved in the SF-B3LYP description of the lowest singlet state of the end-on complex $\left[\mathrm{Cu}(\text { trenMe } 3)\left(\mathrm{O}_{2}\right)\right]^{+}$.

Initially developed within the SCF or the CIS(D) frameworks, ${ }^{50,51}$ the SF approach has been merged with DFT through time-dependent density functional theory within the Tamm-Dancoff approximation, ${ }^{54-56}$ a procedure that has been successfully applied to the description of (poly)radicals, ${ }^{54,56,63}$ and to that of some transition-metal compounds. ${ }^{64-66}$ A detailed discussion of the differences occurring between TD-DFT and SF-(TD)DFT can be found in Refs. 54-56 and will not be developed further here.

\section{B. Computational details}

The DZVP2 basis set has been employed for all atoms. ${ }^{67}$ A set of $p$ diffuse functions was added on oxygen and nitrogen. ${ }^{68,69}$

Except for Sec. III C, the geometries considered to evaluate the adiabatic singlet-triplet gaps come from B3LYP optimizations. $^{57}$

The CASSCF and CASMP2 calculations were done according to the scheme implemented in GAUSSIAN03. ${ }^{70,71}$ The notation $(n, m)$ will be used to label an active space characterized by $n$ electrons in $m$ active orbitals.

The QCHEM 2.1 package was used to run all DFT com- 


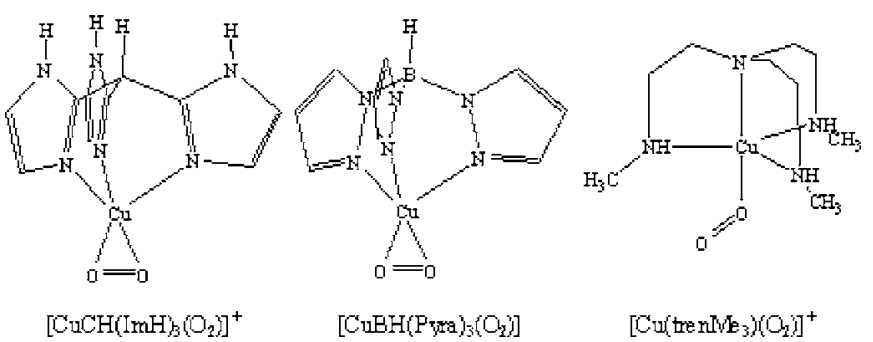

FIG. 2. Three copper-dioxygen adducts investigated in this paper that span various chemical situations encountered when modeling mononuclear copper sites.

putations, including the SF ones that were considered within the time-dependent DFT framework. ${ }^{54,72}$ The following functionals have been used: BP86, ${ }^{73,74} \mathrm{BLYP}^{73,75,76} \mathrm{~B}^{2} \mathrm{LYP}^{77}$ $\left[20 \% \mathrm{HF}+8 \%\right.$ Slater $^{78}+72 \%$ Becke $^{73}$ for exchange and $19 \%$ $\mathrm{VWN}^{79}+81 \% \mathrm{LYP}^{75,76}$ for correlation], and the 50/50 functional $(50 \%$ Hartree-Fock $+8 \%$ Slater $+42 \%$ Becke for exchange and $19 \% \mathrm{VWN}+81 \%$ LYP for correlation). Note that the three later functionals exhibit an increasing weight of exact Hartree-Fock exchange for a given correlation functional. The calculations have been supplemented by results obtained considering the B3PW91 functional. ${ }^{77,80-82}$ B3LYP is certainly the most widely used functional nowadays, although not perfect, ${ }^{83}$ but B3PW91 is sometimes preferred in some cases. $^{84-87}$

\section{RESULTS, DISCUSSION, AND APPLICATIONS}

\section{A. Validation}

$\left[\mathrm{CuCH}(\mathrm{ImH})_{3}\left(\mathrm{O}_{2}\right)\right]^{+}$and $\left[\mathrm{CuBH}(\text { Pyra })_{3}\left(\mathrm{O}_{2}\right)\right]$. We first consider the two side-on adducts depicted on Fig. 2. Both $\left[\mathrm{CuCH}(\mathrm{Im} \mathrm{H})_{3}\right]^{+}$and $\left[\mathrm{CuBH}(\text { Pyra })_{3}\right]$ bind $\mathrm{O}_{2}$ side-on in either a triplet or a singlet state. Table I gathers the singlet- triplet gaps defined as $E(S)-E(T)$, obtained from DFT, both in the restricted or unrestricted formalisms, together with those given by multideterminantal methods. The active spaces for $\operatorname{CASSCF}(18,12)$ and $\operatorname{CASSCF}(12,8)$ can be, respectively, schemed as ${ }^{57}$

$$
\begin{aligned}
& {\left[(3 d)^{10} 4 s^{0}\left(\sigma, \sigma^{*}\right)^{2}\left(\pi_{x}, \pi_{y} \pi_{x}^{*}, \pi_{y}^{*}\right)^{6}\right],} \\
& {\left[(3 d)^{4}\left(\sigma, \sigma^{*}\right)^{2}\left(\pi_{x}, \pi_{y}, \pi_{x}^{*}, \pi_{y}^{*}\right)^{6}\right] .}
\end{aligned}
$$

The $\sigma$ and $\pi$ orbitals are related to the $\mathrm{O}_{2}$ moiety and the $3 d$ and $4 s$ orbitals to the metal cation: the five $3 d$ orbitals are considered in the first case, only two are retained in the second case.

For $\left[\mathrm{CuCH}(\mathrm{ImH})_{3}\left(\mathrm{O}_{2}\right)\right]^{+}$the $\mathrm{CASMP} 2(18,12)$ value $(-4.62 \mathrm{kcal} / \mathrm{mol})$ for the singlet-triplet gap will be taken as the reference. For $\left[\mathrm{CuBH}(\mathrm{Pyra})_{3}\left(\mathrm{O}_{2}\right)\right]$, the reference will be the experimental value of $-4.29 \pm 0.86 \mathrm{kcal} / \mathrm{mol}$ derived from magnetochemistry measurements on a very closely related complex. ${ }^{58}$ This value is close to that provided by a CASSCF $(12,8)$ computation $(-4.28 \mathrm{kcal} / \mathrm{mol}) .{ }^{57}$

As anticipated, the restricted formalism R-DFT (R-B3LYP) fails to properly evaluate the singlet-triplet gaps for $\left[\mathrm{CuCH}(\mathrm{ImH})_{3}\left(\mathrm{O}_{2}\right)\right]^{+}$and $\left[\mathrm{CuBH}(\mathrm{Pyra})_{3}\left(\mathrm{O}_{2}\right)\right]$ : for these two oxygenated adducts, the singlet states are always under stabilized due to the intrinsic monodeterminantal character of the restricted wave functions.

Using broken symmetry (BS-UB3LYP) for $\left[\mathrm{CuCH}(\mathrm{ImH})_{3}\left(\mathrm{O}_{2}\right)\right]^{+}$improves the energy of the singlet states and reduces the gap with respect to the restricted approach. It now amounts to $7.03 \mathrm{kcal} / \mathrm{mol}$. Nevertheless, this value remains far from those issued from CASSCF or CASMP2 computations, namely, -6.94 and $-4.62 \mathrm{kcal} / \mathrm{mol}$, respectively.

TABLE I. Adiabatic singlet/triplet gaps $(\mathrm{kcal} / \mathrm{mol}): E(S)-E(T)$. The active spaces $(n, m)$ generate the following number of determinants for a singlet state: 6 for $(4,3), 10$ for $(6,4), 406$ for $(12,8)$, and 48400 for $(18,12)$.

\begin{tabular}{lccc}
\hline \hline & {$\left[\mathrm{CuCH}(\mathrm{ImH})_{3}\left(\mathrm{O}_{2}\right)\right]^{+}$} & {$\left[\mathrm{CuBH}(\mathrm{Pyra})_{3}\left(\mathrm{O}_{2}\right)\right]$} & $\left.\left[\mathrm{Cu}(\operatorname{trenMe})_{3}\right)\left(\mathrm{O}_{2}\right)\right]^{+}$ \\
\hline Binding mode & Side-on & Side-on & End-on \\
R-B3LYP & 14.31 & 9.20 & 19.67 \\
BS-UB3LYP & 7.03 & $\ldots{ }^{\mathrm{a}}$ & 14.09 \\
SF-BP86 & -18.03 & -19.85 & -5.26 \\
SF-BLYP & -16.93 & -17.66 & -4.50 \\
SF-B3LYP & -2.44 & -4.37 & 8.21 \\
SF-B3PW91 & -3.34 & -5.36 & 7.75 \\
SF-50/50 & 7.36 & 5.10 & 4.73 \\
CASSCF $(4,3)$ & -2.93 & -0.45 & $\ldots$ \\
CASMP2 $(4,3)$ & -8.21 & -6.06 & $\ldots$ \\
CASSCF $(6,4)$ & $\ldots$ & $\ldots$ & -2.07 \\
CASMP2 $(6,4)$ & $\ldots$ & $\ldots$ & 5.98 \\
CASSCF $(12,8)$ & $\ldots$ & $-4.28^{\mathrm{b}}$ & $\ldots$ \\
CASSCF $(18,12)$ & $-6.94^{\mathrm{b}}$ & $\ldots$ & -0.49 \\
CASMP2 $(18,12)$ & $-4.62^{\mathrm{b}}$ & $\ldots$ & $\ldots$ \\
Experimental & $\cdots$ & $-4.29 \pm 0.86^{\mathrm{c}}$ & $\ldots$ \\
\hline
\end{tabular}

${ }^{\mathrm{a}}$ All attempts to obtain the broken-symmetry energy failed as the restricted wave function was systematically recovered.

${ }^{\mathrm{b}}$ From Ref. 57.

${ }^{\mathrm{c}}$ From Ref. 58. Gap reported for the related $\left[\mathrm{CuTp}{ }^{\mathrm{Ad}, i \mathrm{Pr}}\left(\mathrm{O}_{2}\right)\right]$ complex. Tp ${ }^{\mathrm{Ad}, i \mathrm{Pr}}$ stands for tris (3-adamantly-5isopropyl-1-pyrazoly)hydroborate. 
We now turn to the SF-DFT results. They exhibit a strong functional dependence. Using BP86, the singlet-triplet gaps amount to -18.03 and to $-19.85 \mathrm{kcal} / \mathrm{mol}$ for $\left[\mathrm{CuCH}(\mathrm{ImH})_{3}\left(\mathrm{O}_{2}\right)\right]^{+}$and $\left[\mathrm{CuBH}(\mathrm{Pyra})_{3}\left(\mathrm{O}_{2}\right)\right]$, respectively. BLYP provides very similar values. Both these nonhybrid functionals clearly overstabilize the singlet states with respect to the reference values or to experimental data.

At variance, the 50/50 functional seems to favor the triplet states. For both adducts, this overstabilization leads to locate the triplet states as the fundamental, in opposite to CASSCF/CASMP2 computations and to the available experimental data.

The other hybrid functionals provide, however, clearly better balanced results. For $\left[\mathrm{CuCH}(\mathrm{ImH})_{3}\left(\mathrm{O}_{2}\right)\right]^{+}$, the gaps obtained amount to $-2.44 \mathrm{kcal} / \mathrm{mol}$ (SF-B3LYP) and to $-3.34 \mathrm{kcal} / \mathrm{mol}$ (SF-B3PW91), thus predicting the singlet coordination to be the most favorable and providing values within the same range of energy as those obtained from the CASMP2 $(18,12)$ calculation: ${ }^{57}-4.62 \mathrm{kcal} / \mathrm{mol}$.

For $\left[\mathrm{CuBH}(\mathrm{Pyra})_{3}\left(\mathrm{O}_{2}\right)\right]$, the SF-hybrid-DFT computations also provide good qualitative and quantitative predictions. The singlet state is stabilized by $-4.37 \mathrm{kcal} / \mathrm{mol}$ (SF-B3YLP) or by $-5.36 \mathrm{kcal} / \mathrm{mol}$ (SF-B3PW91) with respect to the triplet state. Such values are in almost perfect agreement with those obtained either from $\operatorname{CASSCF}(12,8)$ computations $(-4.28 \mathrm{kcal} / \mathrm{mol})^{57}$ or from experiment $(-4.29 \pm 0.86 \mathrm{kcal} / \mathrm{mol}) .^{58}$

$\left[\mathrm{Cu}\left(\text { tren } \mathrm{Me}_{3}\right)\left(\mathrm{O}_{2}\right)\right]^{+}$. The third adduct investigated, $\left[\mathrm{Cu}\left(\text { tren } \mathrm{Me}_{3}\right)\left(\mathrm{O}_{2}\right)\right]^{+}$, completes the series and provides an example of end-on coordination (Fig. 2). For that system, no experimental data are available, and all attempts to run the CASMP2 $(18,12)$ perturbation felt due to a strong divergence of the perturbation part, a feature sometimes encountered. ${ }^{88}$

Both R-B3LYP and BS-UB3LYP gaps appear unrealistic: a value of $14.09 \mathrm{kcal} / \mathrm{mol}$ is recovered at the latter level, far from the $-0.49 \mathrm{kcal} / \mathrm{mol}$ provided by the CASSCF $(18,12)$ computations. The SF approach provides results that are more balanced. However, both SF-BP86 and SF-BLYP predict a singlet ground state (-5.26 and $-4.50 \mathrm{kcal} / \mathrm{mol}$,

respectively), whereas increasing the exact Hartree-Fock exchange by using either SF-B3LYP, SF-B3PW91, or SF$50 / 50$ favors the triplet state $(8.21,7.75$, and $4.73 \mathrm{kcal} / \mathrm{mol}$ respectively). If we follow the tendencies observed previously for $\left[\mathrm{CuCH}(\mathrm{ImH})_{3}\left(\mathrm{O}_{2}\right)\right]^{+}$and $\left[\mathrm{CuBH}(\mathrm{Pyra})_{3}\left(\mathrm{O}_{2}\right)\right]$ we should trust these three calculations and conclude that the triplet state is the ground state for that species, and that the singlet-triplet gap amounts to several $\mathrm{kcal} / \mathrm{mol}$. Another argument, external, supports that conclusion. The singlet or triplet character of a number of various end-on $\mathrm{O}_{2}$ adducts has been reported recently. ${ }^{35}$ For an anionic set of ligands, the singlet-triplet gaps were in a range of 3-5 kcal/mol. For a neutral ligand set $\left(\mathrm{N}_{3}\right)\left(\mathrm{H}_{2} \mathrm{O}\right)$, the gaps even rose to $9.19 \mathrm{kcal} / \mathrm{mol}$, still in favor of a triplet ground state. Our SF-hybrid-DFT results appear thus fully consistent with this work. Corroboration will be provided in Sec. III D.

Preliminary conclusion. Gathering all SF-DFT calculations reveals a dependency of the $T / S$ gap with respect to the percentage of exact exchange in the functional used: increas- ing the weight of that quantity favors the triplet state. Such a behavior has been reported previously when using the standard DFT approach. ${ }^{58}$

Nevertheless, we have studied three chemically different species, providing examples of neutral or cationic, aliphatic, or aromatic precursory copper (I) complexes binding $\mathrm{O}_{2}$ in a side-on or end-on mode. It appears that the SF-DFT methodology provides a quantitative and trustworthy energetics when compared to experimental or large-size MRPT gaps if using the B3LYP or B3PW91 functionals. It thus represents a clear alternative to the broken-symmetry approach that fails to describe triplet-singlet gaps in mononuclear copperdioxygen complexes exhibiting a strong superoxide character, ${ }^{8}$ or to the highly CPU-demanding MRPT or MRCI approaches.

\section{B. Spin contamination}

The BS-UDFT calculations performed on the singlet states exhibit a high spin contamination. $\left\langle S^{2}\right\rangle$ amounts to about 0.6 for both $\left[\mathrm{CuCH}(\mathrm{ImH})_{3}\left(\mathrm{O}_{2}\right)\right]^{+}$and $\left[\mathrm{Cu}\left(\text { trenMe } \mathrm{Me}_{3}\right)\left(\mathrm{O}_{2}\right)\right]^{+}$. Such large values of spin contamination for singlet BS wave functions describing copper-dioxygen adducts have already been observed. ${ }^{8}$ Of course, $\left\langle S^{2}\right\rangle$ computed with the single $\mathrm{KS}$ determinant and the $\mathrm{KS}$ orbitals is not a true measure of the exact $\left\langle S^{2}\right\rangle$ of the DFT density ${ }^{31}$ as $\left\langle S^{2}\right\rangle$ cannot be evaluated from the sole one-particle density due to the bielectronic nature of the operator. We moreover recall that the spin contamination is not a usable indicator to evaluate the quality of unrestricted wave functions: ${ }^{89,90}$ larger values of $\left\langle S^{2}\right\rangle$ than those expected indicate that the improvement of the energetic is, at least partly, an intrinsic artifact. Keeping these limitations in mind, we note, however, that the SF-B3LYP and SF-B3PW91 wave functions are not highly spin contaminated. The highest spin contamination for the singlet states was obtained for $\left[\mathrm{Cu}\left(\text { tren } \mathrm{Me}_{3}\right)\left(\mathrm{O}^{2}\right)\right]^{+}$and never exceeded 0.15. As we have performed the SF-DFT computations using restricted orbitals, the spin contamination invoked in these calculations originates from a completely different reason than those implied in unrestricted formalisms. Here, such a small contamination might be explained in terms of spin completeness of the excitation set spanned by the SF formalism considered. In the presently used framework of SF-DFT, which involves only single excitations, the set of excitations does not span a complete spin set. As seen from Fig. 1 (top), configurations $b$ $\left(1^{\beta} 2^{\alpha \beta} 3^{\alpha}\right)$ and $c\left(1^{\beta} 2^{\alpha} 3^{\alpha \beta}\right)$ are not balanced by their spin counterparts $\left(1^{\alpha} 2^{\alpha \beta_{3}} 3^{\beta}\right)$ and $\left(1^{\alpha} 2^{\beta} 3^{\alpha \beta}\right)$ since these two configurations are not reachable by spin-flip excitations from the triplet reference. This results in a spin polarization that may lead to the contamination of the wave function. ${ }^{91}$ In practice, at least for the cases investigated here, the non-spin-complete configurations, responsible for spin contamination, have a nondominant weight in the full SF-DFT wave function: this explains why the departure of $\left\langle S^{2}\right\rangle$ from its expected value is weak and why spin contamination does not strongly affect the energies. 
TABLE II. Geometry optimization of singlet $\left[\mathrm{Cu}(\operatorname{tren})\left(\mathrm{O}_{2}\right)\right]^{+}$. Distances are in $\AA$ and angles in degrees. $\Delta E_{\text {opt }}$ is the energy gain during the SF optimization $(\mathrm{kcal} / \mathrm{mol})$. The end-on structure is lower by $2.2 \mathrm{kcal} / \mathrm{mol}$ than the side-on one.

\begin{tabular}{lccccc}
\hline \hline & \multicolumn{2}{c}{ Side-on } & & \multicolumn{2}{c}{ End-on } \\
\cline { 2 - 3 } \cline { 5 - 6 } & R-B3LYP & SF-B3LYP & & R-BLYP & SF-B3LYP \\
\hline$\Delta \mathrm{E}_{\text {opt }}$ & $\ldots$ & -0.05 & & $\ldots$ & -0.13 \\
$\mathrm{Cu}-\mathrm{O}$ & 1.917 & 1.913 & & 1.893 & 1.883 \\
$\mathrm{O}-\mathrm{O}$ & 1.321 & 1.322 & & 1.295 & 1.309 \\
$\mathrm{Cu}-\mathrm{N}$ & $2.291 / 2.065$ & $2.297 / 2.068$ & & 2.135 & $2.132^{\mathrm{a}}$ \\
$\mathrm{Cu}-\mathrm{N}_{\mathrm{ap}}$ & 2.711 & 2.697 & & 2.179 & 2.163 \\
$\mathrm{CuOO}$ & 70.0 & 69.4 & & 106.0 & 106.2 \\
\hline \hline
\end{tabular}

${ }^{\mathrm{a}}$ Averaged over the three equatorial $\mathrm{Cu}-\mathrm{N}$ bond lengths.

\section{Application: Geometry optimizations}

In many cases and works, a general procedure to deal with the singlet-triplet gap of the copper-dioxygen adducts is to consider geometries optimized at the restricted or unrestricted DFT levels. Then, single point calculations (MRCI, MRPT, or other) can be performed to get reliable adiabatic or vertical gaps. A more proper treatment would, in fact, require the geometry optimizations to be performed at the multireference level. However, CASSCF optimized geometries have been shown to be less reliable than the DFT ones ${ }^{8}$ and CASMP2 optimizations are, most of the time, not reachable for systems of such size (a few systems of small size have been reported). ${ }^{47,92}$ There is, however, no alternative to using DFT for geometry optimizations and it has always been considered that using restricted DFT and even using unrestricted DFT would provide useable geometries for the systems investigated here or their analogs. ${ }^{35,57,93,94}$

We will take advantage of the appealing possibility of SF-DFT to optimize such adducts. In order to explore this point, we here have considered the $\left[\mathrm{Cu}(\operatorname{tren})\left(\mathrm{O}_{2}\right)\right]^{+}$adduct in its two possible coordination modes, end-on and side-on. We first have optimized the structures of the singlet states at the R-B3LYP level, and then the optimizations were performed at the SF-B3LYP level using the R-B3LYP structures as preoptimized structures. Some essential structural parameters are reported in Table II.

In both the side-on and the end-on coordination modes, the SF-DFT optimizations of the singlet states do not lead to any drastic geometric changes with respect to the R-B3LYP geometries. This is, globally, corroborated by the small energetic gains achieved within the optimization procedures. Such a result thus comforts the fact that R-DFT optimized singlet states can be considered as reliable. In the present case, there is a clear explanation for that point. As seen from the inspections of the SF-B3LYP wave functions (Fig. 1), the weight of the leading, close-shell, determinant amounts to $90 \%$ for the side-on adduct, and still amounts to $77 \%$ for the end-on adduct. As far as the leading determinant(s) will be close-shell, R-DFT optimizations appear reliable. However, in view of the weak increase of computational costs turning from R-DFT to SF-DFT, the later approach may be encouraged. The same conclusions can be drawn for the determination of vibrational frequencies. ${ }^{95}$

\section{Application: designing zeroth-order variational spaces for $\mathrm{MRCl}$ or MRPT calculations}

In this last section, we will present another appealing application of SF-DFT computations, namely, using the SFDFT procedure as a potentially powerful tool to build reference CASSCF or MCSCF spaces designed to further MRCI or MRPT treatments. Indeed, the electrons and the set of molecular orbitals, occupied and virtual, involved in the excitations appearing in the SF-DFT wave functions can be expected to span a minimal relevant variational active space. If we focus on CASSCF computations, there are three usual ways of building active spaces: (a) considering the highest $M$ occupied orbitals, the $n$ associated electrons, and the $M^{\prime}$ lowest virtual orbitals; (b) considering the natural occupations of the occupied and virtual orbitals; (c) considering chemically meaningful orbitals, namely, those orbitals, the occupied and the corresponding virtual ones, associated with electrons involved into the chemical or physicochemical processes investigated. Approach (a) implies some arbitrariness; it is also risky as the orbital energy sorting within the whole set of orbitals can be extremely dependent, especially for the virtual ones, on the way they have been initially obtained. Approach (b) very certainly is the most efficient in order to get the lowest total energy of the system under study; it suffers the drawback that determining natural occupation numbers prerequires a large-size MCSCF, CASSCF, or CI calculation. Approach (c) certainly remains the most chemically satisfying one as it is expected to ensure the continuity of the active space with respect to any previously analyzed chemical or physicochemical process. ${ }^{96,97}$ It has the drawback not necessarily being friendly to the variation theorem: those orbitals and electrons considered as chemically relevant are not necessarily those that will help in decreasing the CASSCF energy. A commonly encountered problem comes from lone pairs. ${ }^{98,99}$

None of these approaches can thus be considered as a panacea. Consequently, any way of designing an efficient but "small enough" zeroth-order variational space for further MRCI or MRPT calculations seems thus welcomed. Indeed, some purely $a b$ initio approaches have already tackled that problem. ${ }^{100-106}$ In this section, we will illustrate how SFDFT computations can help in designing such a space. The starting idea is very simple: as SF-DFT computations are not the limiting CPU-time step if aiming at performing a pure high-level ab initio multireference correlated computation, then, let us first perform a SF-DFT computation and retain in the zeroth-order space to be built only those electrons and orbitals involved in the final SF-DFT wave functions. We have here applied this idea to the three dioxygen adducts investigated previously.

For the side-on coordination, the SF excitations lead to consider the CASSCF space spanned by four electrons in three orbitals (Fig. 1, top), which results in a six-determinant expansion for the singlet state, for example, to be compared to the 48400 determinant basis for the $(18,12)$ wave function or to the 406 determinant expansion generated by the $(12,8)$ space. The singlet state is then predicted (CASMP2) to be lower than the triplet by $8.21 \mathrm{kcal} / \mathrm{mol}$ for $\left[\mathrm{CuCH}(\operatorname{ImH})_{3}\left(\mathrm{O}_{2}\right)\right]^{+}$: this result is consistent with both the 
CASSCF $(18,12)$ and CASMP2 $(18,12)$ calculations. We also notice a full agreement between the two CASSCF wave functions, namely, $(4,3)$ and $(18,12)$ in terms of the leading configurations. These configurations are also those provided by the SF computations. For the $\left[\mathrm{CuBH}(\mathrm{Pyra})_{3}\left(\mathrm{O}_{2}\right)\right] \mathrm{com}$ plex, the situation is similar: the gaps obtained from a CASMP2 $(4,3)$ computation amounts to $-6.06 \mathrm{kcal} / \mathrm{mol}$, close to CASSCF $(12,8)$ value of $-4.28 \mathrm{kcal} / \mathrm{mol}$ (Ref. 57) and to the experimental gap $(-4.29 \pm 0.86 \mathrm{kcal} / \mathrm{mol})$ (Ref. 58) obtained for the closely related substituted complex $\left[\mathrm{CuTp}{ }^{\operatorname{Ad}, \mathrm{iPr}}\left(\mathrm{O}_{2}\right)\right]$. The end-on adduct observed with the $\left[\mathrm{Cu}\left(\text { trenMe } \mathrm{Me}_{3}\right)\left(\mathrm{O}_{2}\right)\right]^{+}$complex behaves as well (Fig. 1, bottom). The SF-driven CASMP2 $(6,4)$ computation predicts the triplet state to be more stable than the singlet by $5.98 \mathrm{kcal} / \mathrm{mol}$. This value is in nice agreement with our SFDFT results and with recent $a b$ initio calculations, ${ }^{35}$ in which end-on structures are found to have a triplet ground state associated with $S / T$ gaps amounting to a few $\mathrm{kcal} / \mathrm{mol}$. For the singlet state, the $(6,4)$ wave function expands over ten determinants only.

These results fully validate using SF-DFT main excitations to design drastically reduced active spaces. Being smaller, such variational spaces are more controllable and the second-order perturbation corrections that can be applied next may be more tractable and less subject to unpredictable divergences. Moreover, this way of doing avoids the chemical models to be dramatically simplified.

\section{CONCLUSIONS}

In this work, we have applied the spin-flip approach within the framework of time-dependent-density-functionaltheory to calculate singlet-triplet gaps in copper (I) dioxygen adducts taken as well-documented examples. A very interesting alternative to more classical multiconfigurational approaches has emerged.

For side-on complexes, SF-DFT coupled to the B3LYP or B3PW91 functionals predicts the same relative stability as the more expensive CASSCF or CASMP2 computations or as the experimental results. For end-on complexes, the method is also in good agreement with the three functionals considered, namely, 50-50, B3LYP and B3PW91. The approach allows recovering the triplet state as the ground state.

Although the possibility of using SF computations to avoid a broken-symmetry framework and large-scale multireference computations is intrinsically promising, our results point out for some clear-cut added value of the SF approach. First, it can be used to perform "CPU-cheap" geometry optimizations on large multireference systems, and consequently frequency calculations, taking into account the multideterminantal character of the systems investigated. Second, the leading excitations of the SF wave functions can be considered in designing reduced active spaces if pure $a b$ initio computations (MRCI or MRPT) are required.

\section{ACKNOWLEDGMENTS}

The CASSCF and CASMP2 calculations have been performed at the CRIHAN regional supercomputing center (F. 76800 Saint-Etienne-du-Rouvray, France). The authors greatly acknowledge H. Gérard (LCT) and J.-P. Piquemal (LCT) for stimulating discussions, together with L.-H. Jolly (LCT) for technical assistance.

${ }^{1}$ W. Kohn, Rev. Mod. Phys. 71, 1253 (1998).

${ }^{2}$ S. Niu and M. B. Hall, Chem. Rev. (Washington, D.C.) 100, 353 (2000).

${ }^{3}$ J. F. Harrison, Chem. Rev. (Washington, D.C.) 100, 679 (2000).

${ }^{4}$ J. Baker and P. Pulay, J. Comput. Chem. 10, 1184 (2003).

${ }^{5}$ N. E. Schultz, Y. Zhao, and D. G. Truhlar, J. Phys. Chem. A 109, 11127 (2005).

${ }^{6}$ F. Furche and J. P. Perdew, J. Chem. Phys. 124, 044103 (2006).

${ }^{7}$ W. Kohn and L. J. Sham, Phys. Rev. 140, A1133 (1965).

${ }^{8}$ B. F. Gherman and C. J. Cramer, Inorg. Chem. 43, 7281 (2004) and supplementary material.

${ }^{9}$ F. Neese, JBIC, J. Biol. Inorg. Chem. 11, 702 (2006) and references therein.

${ }^{10}$ P. E. M. Siegbahn, JBIC, J. Biol. Inorg. Chem. 11, 695 (2006) and references therein.

${ }^{11}$ A. Ghosh, JBIC, J. Biol. Inorg. Chem. 11, 671 (2006); 11, 712 (2006) and references therein.

${ }^{12}$ L. Noodleman and W.-G. Han, JBIC, J. Biol. Inorg. Chem. 11, 674 (2006) and references therein.

${ }^{13}$ J. P. Klinman, J. Biol. Chem. 281, 3013 (2006).

${ }^{14}$ J. P. Klinman, Chem. Rev. (Washington, D.C.) 96, 2541 (2006).

${ }^{15}$ L. C. Stewart and J. P. Klinman, Annu. Rev. Biochem. 57, 551 (1988).

${ }^{16}$ B. A. Eipper, D. A. Stoffers, and R. E. Mains, Annu. Rev. Neurosci. 15, 57 (1992).

${ }^{17}$ B. A. Eipper, S. L. Milgram, E. J. Husten, H. Y. Yun, and R. E. Mains, Protein Sci. 2, 489 (1993).

${ }^{18}$ A. Crespo, M. A. Martí, A. E. Roitberg, L. M. Amzel, and D. A. Estrin, J. Am. Chem. Soc. 128, 12817 (2006).

${ }^{19}$ H. Decker, T. Schweikardt, and F. Tuczek, Angew. Chem., Int. Ed. 45, 4546 (2006).

${ }^{20}$ Y. Matoba, T. Kumagai, A. Yamamoto, H. Yoshitsu, and M. Sugiyama, J. Biol. Chem. 281, 8981 (2006).

${ }^{21}$ C. J. Cramer, M. Wloch, P. Piecuch, C. Puzzarini, and L. Gagliardi, J. Phys. Chem. A 110, 1991 (2006).

${ }^{22}$ J. P. Klinman, Chem. Rev. (Washington, D.C.) 96, 2541 (1996).

${ }^{23}$ E. I. Solomon, U. M. Sundaram, and T. E. Machonkin, Chem. Rev. (Washington, D.C.) 96, 2563 (1996).

${ }^{24}$ L. M. Mirica, X. Ottenwaelder, and T. D. P. Stack, Chem. Rev. (Washington, D.C.) 104, 1013 (2004).

${ }^{25}$ S. Schindler, Eur. J. Inorg. Chem. 11, 2311 (2000).

${ }^{26}$ L. Noodleman and D. A. Case, Adv. Inorg. Chem. 38, 423 (1992).

${ }^{27}$ L. Noodleman, C. Y. Peng, D. A. Case, and J.-M. Mouesca, Coord. Chem. Rev. 144, 199 (1995).

${ }^{28}$ V. N. Staroverov and E. R. Davidson, J. Am. Chem. Soc. 122, 186 (2000).

${ }^{29}$ D. A. Hrovat, J. A. Duncan, and W. T. Borden, J. Am. Chem. Soc. 121, 169 (1999).

${ }^{30}$ E. R. Davidson, Chem. Phys. Lett. 284, 301 (1998).

${ }^{31}$ A. A. Jarzecki and E. R. Davidson, J. Phys. Chem. A 102, 4742 (1998).

${ }^{32}$ T. Ziegler, A. Rauk, and E. J. Baerends, Theor. Chim. Acta 43, 261 (1977).

${ }^{33}$ K. Yamagushi, F. Jensen, A. Dorigo, and K. N. Houk, Chem. Phys. Lett. 149, 537 (1988).

${ }^{34}$ H. Isobe, Y. Takano, Y. Kitagawa, T. Kawakami, S. Yamanaka, K. Yamaguchi, and K. N. Houk, Mol. Phys. 100, 717 (2002).

${ }^{35}$ B. F. Gherman, D. E. Heppner, W. B. Tolman, and C. J. Cramer, JBIC, J. Biol. Inorg. Chem. 11, 197 (2006).

${ }^{36}$ P. Chen and E. I. Solomon, J. Am. Chem. Soc. 126, 4991 (2004).

${ }^{37}$ C. Adamo, V. Barone, A. Bencini, R. Broer, M. Filatov, N. M. Harrison, F. Illas, J.-P. Malrieu, and I. de P. R. Moreira, J. Chem. Phys. 124, 107101 (2006).

${ }^{38}$ C. R. Kinsinger, B. F. Gherman, L. Gagliardi, and C. J. Cramer, JBIC, J. Biol. Inorg. Chem. 10, 778 (2005).

${ }^{39}$ C. Daul and A. Goursot, Int. J. Quantum Chem. 29, 779 (1986).

${ }^{40}$ C. Daul, Int. J. Quantum Chem. 52, 867 (1994).

${ }^{41}$ A. C. Stückl, C. A. Daul, and H. U. Güdel, Int. J. Quantum Chem. 61, 579 (1997).

${ }^{42}$ T. Mineva, A. Goursot, and C. Daul, Chem. Phys. Lett. 350, 147 (2001).

${ }^{43}$ M. Atanasov and C. A. Daul, C. R. Chim. 8, 1421 (2005).

${ }^{44}$ A. Borel and C. A. Daul, J. Mol. Struct.: THEOCHEM 762, 93 (2006). 
${ }^{45}$ See, for example: $A b$ Initio Methods in Quantum Chemistry, Advances in Chemical Physics, edited by K. P. Lawley (Wiley, New York, 1987), Pts. I and II.

${ }^{46}$ Recent Advances in Multireference Methods, edited by K. Hirao (World Scientific, Singapore, 1999).

${ }^{47}$ M. F. Rode and H.-J. Werner, Theor. Chem. Acc. 114, 309 (2005).

${ }^{48}$ For an example: J.-Y. Hasegawa, K. Pierloot, and B. O. Roos, Chem. Phys. Lett. 335, 503 (2001).

${ }^{49}$ For example, modeling an imidazole moiety, a $\sigma$-donor and $\pi$-acceptor ligand, by $\mathrm{NH}_{3}, \sigma$ donor only, can lead to severe artifacts. See the case of tyrosinase: J.-P. Piquemal and J. Pilmé, J. Mol. Struct.: THEOCHEM 764, 77 (2006).

${ }^{50}$ A. I. Krylov, Chem. Phys. Lett. 338, 375 (2001).

${ }^{51}$ A. I. Krylov, Chem. Phys. Lett. 350, 522 (2001).

${ }^{52}$ A. I. Krylov and C. D. Sherrill, J. Chem. Phys. 116, 3194 (2002).

${ }^{53}$ L. V. Slipchenko and A. I. Krylov, J. Chem. Phys. 117, 3694 (2002).

${ }^{54}$ Y. Shao, M. Head-Gordon, and A. I. Krylov, J. Chem. Phys. 118, 4807 (2003).

${ }^{55}$ F. Wang and T. Ziegler, J. Chem. Phys. 121, 12191 (2004).

${ }^{56} \mathrm{~F}$. Wang and T. Ziegler, J. Chem. Phys. 122, 074109 (2005).

${ }^{57}$ A. de la Lande, H. Gérard, V. Moliner, G. Izzet, O. Reinaud, and O Parisel, JBIC, J. Biol. Inorg. Chem. 11, 593 (2006).

${ }^{58}$ P. Chen, D. E. Root, C. Campochiaro, K. Fujisawa, and E. I. Solomon, J. Am. Chem. Soc. 125, 466 (2003).

${ }^{59}$ N. W. Aboelella, B. F. Gherman, L. M. R. Hill, J. T. York, N. Holm, V. G. Young, Jr., C. J. Cramer, and W. B. Tolman, J. Am. Chem. Soc. 128 3445 (2006).

${ }^{60}$ M. Schatz, V. Raab, S. P. Foxon, J. Brem, S. Schneider, M. Reiher, M. C. Holthausen, J. Sundermeyer, and S. Schindler, Angew. Chem., Int. Ed. 43, 4360 (2004).

${ }^{61}$ A. J. Johansson, M. R. A. Blomberg, and P. E. R. Siegbahn, Inorg. Chem. 45, 1491 (2006).

${ }^{62}$ Note that the adjective "excited" here refers to electronic excitations from the reference configuration, not necessarily from the true ground state.

${ }^{63}$ J. Guan, F. Wang, T. Ziegler, and H. Cox, J. Chem. Phys. 125, 044314 (2006).

${ }^{64}$ F. Wang, T. Ziegler, E. van Lenthe, S. van Gisbergen, and E. J. Baerends, J. Chem. Phys. 122, 204103 (2005).

${ }^{65}$ F. Wang and T. Ziegler, J. Chem. Phys. 123, 154102 (2005).

${ }^{66}$ F. Wang and T. Ziegler, J. Chem. Phys. 123, 194102 (2005).

${ }^{67}$ N. Godbout, D. R. Salahub, J. Andzelm, and E. Wimmer, Can. J. Chem. 70, 560 (1992).

${ }^{68}$ R. Krishnam, J. S. Binkley, R. Seeger, and J. A. Pople, J. Chem. Phys. 72, 650 (1980)

${ }^{69}$ P. M. W. Gill, B. G. Johnson, J. A. Pople, and M. J. Frisch, Chem. Phys. Lett. 197, 499 (1992).

${ }^{70}$ M. J. Frisch, G. W. Trucks, H. B. Schlegel et al., GAUSSIAN 03, Revision A.1, Gaussian, Inc., Pittsburgh, PA, 2003.

${ }^{71}$ J. J. McDouall, K. Peasley, and M. A. Robb, Chem. Phys. Lett. 148, 183 (1988).

${ }^{72}$ J. Kong, C. A. White, A. I. Krylov et al., Q-CHEM 2.0, a high-performance ab initio electronic structure program; J. Comput. Chem. 21, 1532 (2001).

${ }_{74}^{73}$ A. D. Becke, Phys. Rev. A 38, 3098 (1998).

${ }^{74}$ J. P. Perdew, Phys. Rev. B 33, 8822 (1986).
${ }^{75}$ C. Lee, W. Yang, and R. G. Parr, Phys. Rev. B 37, 785 (1988).

${ }^{76}$ B. Miehlich, A. Savin, H. Stoll, and H. Preuss, Chem. Phys. Lett. 157, 200 (1989).

${ }^{77}$ A. D. Becke, J. Chem. Phys. 98, 5648 (1993).

${ }^{78}$ J. C. Slater, Quantum Theory of Molecules and Solids, The SelfConsistent Field for Molecules and Solids (McGraw-Hill, New York, 1974), Vol. 4

${ }^{79}$ S. H. Vosko, L. Wilk, and M. Nusair, Can. J. Chem. 58, 1200 (1980).

${ }^{80}$ J. P. Perdew, in Electronic Structure of Solids '91, edited by P. Ziesche and H. Eschrig (Akademie, Berlin, 1991).

${ }^{81}$ A. D. Becke, J. Chem. Phys. 98, 1372 (1993).

${ }^{82}$ J. P. Perdew and Y. Wang, Phys. Rev. B 45, 13244 (1992).

${ }^{83}$ See, for a recent example, M. D. Wodrich, C. Corminboeuf, and P. von Ragué Schleyer, Org. Lett. 8, 3631 (2006).

${ }^{84}$ T. A. Wesolowski, O. Parisel, Y. Ellinger, and J. Weber, J. Phys. Chem. A 101, 7818 (1997).

${ }^{85}$ H. Gérard, E. Clot, C. Giessner-Prettre, K. G. Caulton, E. R. Davidson, and O. Eisenstein, Organometallics 19, 2291 (2000).

${ }^{86}$ H. Gérard, E. R. Davidson, and O. Eisenstein, Mol. Phys. 100, 533 (2002).

${ }^{87}$ D. Laurencin, R. Villanneau, H. Gérard, and A. Proust, J. Phys. Chem. A 110, 6345 (2006).

${ }^{88}$ P. E. M. Siegbahn, Faraday Discuss. 124, 289 (2003).

${ }^{89}$ P. Cassam-Chenaï and G. S. Chandler, C. R. Acad. Sci. (Paris) II $\mathbf{3 1 4}$ 755 (1992).

${ }^{90}$ P. Cassam-Chenaï and G. S. Chandler, Int. J. Quantum Chem. 46, 593 (1993).

${ }^{91}$ J. S. Sears, C. D. Sherrill, and A. I. Krylov, J. Chem. Phys. 118, 9084 (2003).

${ }^{92}$ J. Hasegawa, K. Pierloot, and B. O. Roos, Chem. Phys. Lett. 335, 503 (2001).

${ }^{93}$ N. W. Aboelella, S. V. Kryatov, B. F. Gherman et al., J. Am. Chem. Soc. 126, 16896 (2004).

${ }^{94}$ C. J. Cramer and R. R. Squires, Org. Lett. 1, 215 (1999).

${ }^{95}$ A. Bérces and T. Ziegler, J. Phys. Chem. 99, 11417 (1995).

${ }^{96}$ O. Parisel and Y. Ellinger, Chem. Phys. 189, 1 (1994).

${ }^{97}$ O. Parisel and Y. Ellinger, Chem. Phys. 205, 323 (1996).

${ }^{98}$ A. D. McLean, B. H. Lengsfield, J. Pacansky, and Y. Ellinger, J. Chem. Phys. 83, 3567 (1985).

${ }^{99}$ O. Parisel, Y. Ellinger, and C. Giessner-Prettre, Chem. Phys. Lett. 250, 178 (1996).

${ }^{100}$ B. Huron, J.-P. Malrieu, and P. Rancurel, J. Chem. Phys. 58, 5745 (1973).

${ }^{101}$ J. Miralles, O. Castell, R. Caballol, and J.-P. Malrieu, Chem. Phys. 172, 33 (1993).

${ }^{102}$ M. Reguero, R. Caballol, J.-L. Heully, and J.-P. Malrieu, Chem. Phys. Lett. 265, 621 (1997).

${ }^{103}$ V. M. García, M. Reguero, and R. Caballol, Theor. Chem. Acc. 98, 50 (1997).

${ }^{104}$ J. Cabrero, N. Ben-Amor, and R. Caballol, J. Phys. Chem. A 103, 6220 (1999).

${ }^{105}$ J. Cabrero, C. de Graaf, E. Bordas, R. Caballol, and J.-P. Malrieu, Chem.Eur. J. 9, 2307 (2003)

${ }^{106}$ J. Pitarch-Ruiz, J. Sanchez-Marin, and D. Maynau, J. Comput. Chem. 23, 1157 (2002) 